This item was submitted to Loughborough's Research Repository by the author.

Items in Figshare are protected by copyright, with all rights reserved, unless otherwise indicated.

\title{
Influence of reduced graphene oxide on the rheological response and chain orientation on shear deformation of high density polyethylene
}

\section{PLEASE CITE THE PUBLISHED VERSION}

http://dx.doi.org/10.1016/j.polymer.2016.01.056

\section{PUBLISHER}

(C) Elsevier

\section{VERSION}

AM (Accepted Manuscript)

\section{PUBLISHER STATEMENT}

This work is made available according to the conditions of the Creative Commons Attribution-NonCommercialNoDerivatives 4.0 International (CC BY-NC-ND 4.0) licence. Full details of this licence are available at: https://creativecommons.org/licenses/by-nc-nd/4.0/

\section{LICENCE}

CC BY-NC-ND 4.0

\section{REPOSITORY RECORD}

Liu, Kangsheng, Efren Andablo-Reyes, Nilesh Patil, Daniel Hermida Merino, Sara Ronca, and Sanjay Rastogi. 2016. "Influence of Reduced Graphene Oxide on the Rheological Response and Chain Orientation on Shear Deformation of High Density Polyethylene". figshare. https://hdl.handle.net/2134/20437. 


\section{Influence of reduced graphene oxide on the rheological response and chain orientation on shear deformation of high density polyethylene}

Kangsheng Liu ${ }^{1}$, Efren Andablo-Reyes ${ }^{1,2}$, Nilesh Patil ${ }^{1}$, Daniel Hermida Merino ${ }^{2,3}$, Sara Ronca $^{1 *}$, Sanjay Rastogi ${ }^{1,3^{*}}$

1. Department of Materials, School of Aeronautical, Automotive, Chemical and Materials Engineering, Loughborough University, Loughborough LE11 3TU, England, U.K.

2. DUBBLE CRG/ESRF, Netherlands Organisation for Scientific Research (NWO), c/o ESRF BP 220, F38043, Grenoble Cedex, France.

3. Dutch Polymer Institute (DPI), P.O. Box 902, 5600AX Eindhoven, The Netherlands.

\section{Abstract}

The rheological response of high density polyethylene/reduced graphene oxide nanoplatelets (HDPE/rGON) composites, and the influence of rGON on chain orientation and crystallization behavior after shear flow are investigated. Melt rheology reveals the presence of strong interaction between polymer chains and the filler. Above $4.0 \mathrm{wt} \%$ of the filler concentration, the terminal region of frequency sweep shows changes in the linear viscoelastic properties of the composites. In particular, at these high concentrations the cross-over frequency at which the transition from predominantly elastic to viscous behavior occurs significantly shifts to lower values, indicating the formation of a solid-like percolated network. A drop in $G$ ' at high frequency $(100 \mathrm{rad} / \mathrm{s})$ is observed in the presence of the filler, and the storage modulus shows minima at filler concentration between 2.0 and $4.0 \mathrm{wt} \%$. The influence of chain-filler interaction 
on chain orientation, and subsequent crystallization behavior after application of shear is followed by time resolved WAXD/SAXS. The orientation of the crystalline domains was quantified by the Herman's orientation factor that supports the presence of strong chain-filler interaction. The Deborah number of reptation and retraction suggests that during the applied nonlinear shear, polymer chains in the composites experience mild stretch that is not significant enough to induce crystallization at the high temperature $\left(136^{\circ} \mathrm{C}\right)$. However, restriction imposed by the filler on the chain mobility is pronounced enough to preserve oriented state that causes anisotropy in crystallization on cooling. The enhanced orientation with increasing filler content is conclusively attributed to the strong chain-filler interaction.

Keywords: HDPE, reduced graphene oxide, WAXD/SAXS

\section{Introduction}

Polymer nanocomposites with improved mechanical, electrical or thermal properties have been investigated intensively, and they have become one of the important classes of materials [1,2]. The change in their properties is intimately related to the aspect ratio, microscopic arrangement of the disperse phase, chain-filler interaction, as well as the formation of filler-filler networks. For example, the presence of filler could significantly influence the polymer chain dynamics, consequently influencing crystallization during shear flow and ultimately mechanical properties of the polymer $[3,4]$. Melt rheological studies have revealed that the presence of nanoparticles in polymers perturbs the microscopic inter-chain arrangement of the polymer network, influencing the linear rheological properties [5]. Understanding the influence of nanofillers on polymer dynamics is crucial for the design and process of nanocomposites with desired properties, for example decrease in entanglement density and ease in processability by incorporating nanofillers 
$[6,7,8,9,10]$. It has also been reported that a strong shear flow, with $D e\left(\tau_{\text {retraction }} \times \dot{\gamma}\right)>1$, plays a crucial role in the polymer chain stretching and orientation, resulting in the formation of highly anisotropic, oriented structures upon crystallization [11,12]. For example, shish-kebabs composed of two distinctive morphologies have been observed under flow condition: the shish structure having large crystal thickness and kebab by relatively thinner folded chain crystals [11]. It is reported in early research on formation of shish-kebab structure during crystallization that the long chain component plays a catalytic role that recruits adjacent chains into the formation of the shish [13]. Important to notice is that in the presence of nanoparticles, the shish-kebab structure that arises from the significant chain stretch and orientation can be enhanced [11]. However, the influence of the filler and filler-chain interaction on the formation of anisotropic structure induced by shear flow remains unclear.

Recently, graphene has attracted considerable attention due to its remarkable electrical, thermal and mechanical properties and its potential in developing high performance nanocomposites $[14,15,16,17]$. Due to its very high aspect ratio [18] and theoretical specific surface area $-2630 \mathrm{~m}^{2} / \mathrm{g}$ [19], graphene is capable of modifying the material properties at very low loadings. For example, $0.028 \mathrm{vol} \%$ of graphene in UHMWPE is reported to produce a composite having electrical conductivity [20]. To obtain graphene or reduced graphene oxide, one of the most commonly used method is thermal reduction of graphene oxide nanoplatelets (GON). It is reported in early research that at temperature as low as $150{ }^{\circ} \mathrm{C}$, GON can be reduced to rGON, as indicated by XPS [21]. In addition, low graphene concentrations could hinder polyethylene chain dynamics causing a drop in complex viscosity [6]. To recall, Liu et al. reported that with increasing graphene content up to $0.8 \mathrm{wt} \%$, the complex viscosity of UHMWPE drops to a minimum value [6]. It is of interest to investigate the influence of molar 
mass, in the presence of graphene, on the rheological response. The drop in viscosity in the presence of filler is of commercial as well as fundamental interest. The reduction in viscosity facilitates the ease in processing of polymers, and demonstrates the significance of chain-filler interaction on morphology development in quiescent as well as flow conditions.

Prior to the reported studies on graphene, the influence of rigid and layered nanoclay particles $[22,23,24,25]$ and high aspect ratio carbon nanotubes (CNTs) $[11,26]$ on the shear induced orientation of polymer composites or composites solutions has been extensively investigated. The orientation of clay/polymer nanocomposites induced by shear has been mainly attributed to the orientation of clays that formed regular structure during shear [22]. CNTs filler is found to be responsible for the chain orientation, that results into the orientated structure of the composites after shear and crystallization [11]. It has also been reported that CNTs could act as shish in the formation of hybrid shish-kebab structure due to its considerably high relaxation time and nucleation efficiency [26].

In this publication, following a two-step preparation method, the HDPE/rGON composites are prepared [6]. To recall, combination of the stability of GON dispersion in water and the ease in reduction of GON by heat treatment allows the homogeneous dispersion of nanosheets in the polymer matrix. The influence of rGON on chain orientation during shear deformation and subsequent crystallization is investigated by time-resolved WAXD/SAXS studies. These findings are linked with linear rheological response of the composites. A comparison with our earlier studies shows the relevance of molar mass on the drop in storage modulus at high frequency.

\section{Experimental}

\subsection{Materials}


Graphene oxide nanosheets (GON) were prepared using a modified Hummers method [6,27]. Materials for the synthesis of GON were purchased from Sigma-Aldrich. HDPE (in the form of fine powder) was purchased from Dow Chemicals. All the reagents were used as received. The estimation of molar mass $\left(M_{\mathrm{w}}\right)$ and molecular weight distribution (MWD) of HDPE was carried out by melt rheology using an Advanced Rheometrics Expansion System (ARES) rheometer. The molar mass parameters were calculated using an Orchestrator software based on refs 28 and 29 and the results are shown in Figure 1.

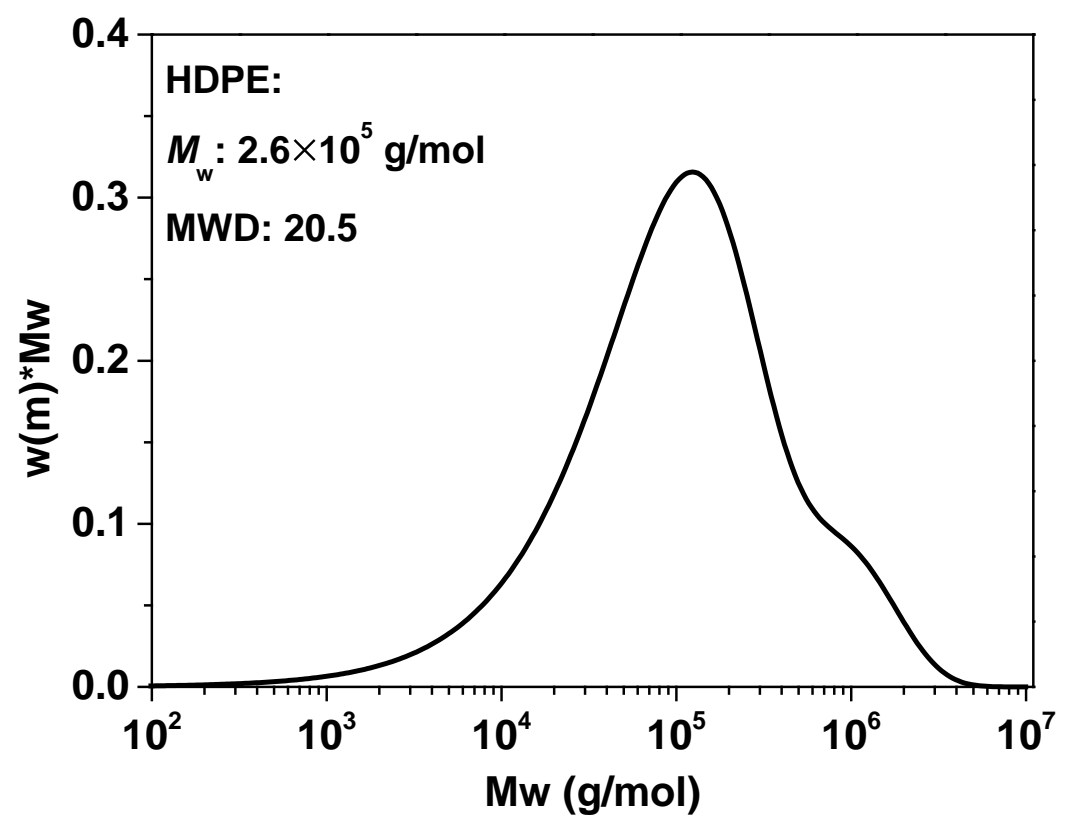

Figure 1. Estimation of $M_{\mathrm{w}}$ and MWD of HDPE by melt rheology.

\subsection{Preparation of HDPE/rGON nanocomposites}

HDPE/rGON nanocomposites were obtained by a two-step preparation method: In Step 1 the required amount of GON was weighed and re-dispersed in $40 \mathrm{ml}$ of water followed by $15 \mathrm{~min}$ ultrasonication, while the required amount of HDPE powder was suspended in acetone and kept 
under magnetic stirring. The ultrasonicated GON water suspension was added to the acetonesuspended HDPE. The mixture was kept under magnetic stirring in a fume cupboard until most of the solvent evaporated and the resultant solid was further dried in a vacuum oven at $40{ }^{\circ} \mathrm{C}$ for at least 12 hours. Due to the hydrophilic character of GON, the use of GON in this step allowed for a better dispersion of the nanoparticles in water. In Step 2, powders prepared in the Step 1 were compression-molded at $160{ }^{\circ} \mathrm{C}$ with pressure reaching 1660 bars in a hydraulic press on a round mold having diameter of $5.0 \mathrm{~cm}$. The heat treatment of the polymer composite allowed the simultaneous reduction of GON to rGON, as described previously in reference 6 . The hydrophobic character of reduced GON over the hydrophilic GON is preferred to ensure a good interaction with the hydrophobic polymer matrix. The compressed reduced composite samples were used for rheological analysis or X-ray characterization. $1.0 \mathrm{wt} \%$ of anti-oxidant (Irganox 1010, Ciba) was added to avoid any possible oxidation or thermal degradation during compression molding. Composites having varying amount of rGON, ranging from $0.0 \mathrm{wt} \%$ to $6.0 \mathrm{wt} \%$, were prepared using the method described above.

\subsection{Characterization of filler and composites}

A JEOL-2000FX Transmission Electron Microscope (TEM) was used to observe the exfoliation state of GON in water with an applied voltage of $200 \mathrm{kV}$. A bench top D2 X-ray Diffraction (XRD) with a radiation wavelength of $\mathrm{CuK}_{\alpha}, 0.154 \mathrm{~nm}$, was used to characterize the

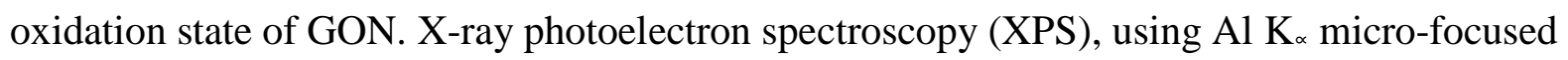
monochromator as X-ray source, was used to characterize the elemental composition of GON and rGON, respectively. The XPS spectra were fitted using Thermo Scientific Avantage data system and Origin software. 
All linear rheological characterizations were performed in an ARES-LS2 rheomoter (TA Instruments) using a $25 \mathrm{~mm}$ parallel plate geometry. Melt state measurements were performed at an isothermal temperature of $160{ }^{\circ} \mathrm{C}$ under nitrogen atmosphere inside a convection oven. A short dynamic time sweep with strain of $0.1 \%$ was performed to reach equilibrium state of the sample and then followed by dynamic frequency sweep test with angular velocities ranging from 0.001 to $100 \mathrm{rad} / \mathrm{s}$ at a constant strain of $0.5 \%$ within the linear viscoelastic regime of the samples.

Time-resolved wide angle X-ray diffraction and small angle X-ray scattering (WAXD/SAXS) experiments were performed on the beamline BM26/DUBBLE at the European Synchrotron Radiation Facility (ESRF) in Grenoble. WAXD patterns were recorded by a Frelon detector placed approximately $89 \mathrm{~cm}$ away from the sample. The wavelength of X-ray applied was 0.103 nm. Background of dark current was subtracted from each WAXD pattern before analysis. SAXS patterns were recorded by a Pilatus detector placed approximately $6.07 \mathrm{~m}$ from the sample. A vacuum chamber was placed in between the detector and the sample to minimize absorption and scattering of X-rays from air. The X-ray wavelength applied was $0.103 \mathrm{~nm}$. A Linkam Shear Cell (CSS-450) equipped with diamond windows was used to perform shear flow experiments followed by the time resolved WAXD/SAXS. The shear cell was placed perpendicular to the incident beam. Two experimental protocols were used to investigate the influence of shear on polymer chains in the presence of rGON, as schematically shown in Scheme 1 and Scheme 2. 


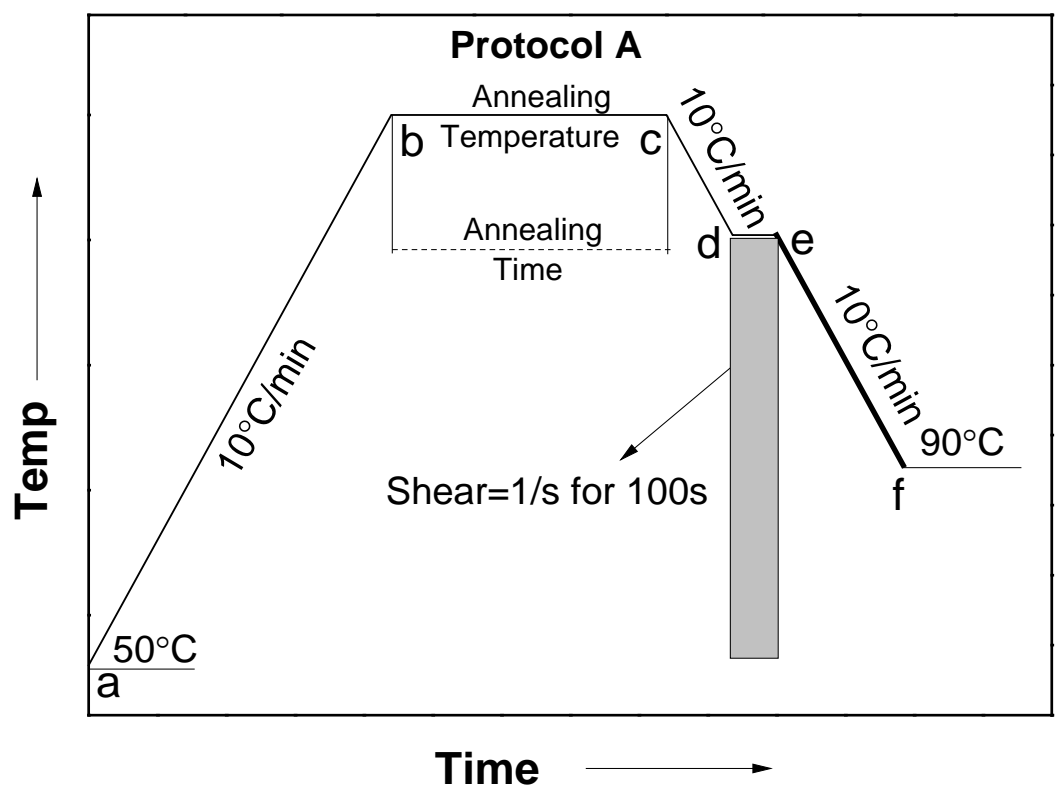

Scheme 1. The schematic representation of shear and temperature profiles during the WAXD/SAXS experiments of Protocol A.

(a-b) Heating from $50{ }^{\circ} \mathrm{C}$ to the annealing temperature of $160{ }^{\circ} \mathrm{C}$ at $10{ }^{\circ} \mathrm{C} / \mathrm{min}$, above the equilibrium melting point $\left(141.5^{\circ} \mathrm{C}\right)$ of linear polyethylene [30];

(b-c) Annealing at $160^{\circ} \mathrm{C}$ for 5 min with the aim to remove sample's thermo-mechanical history;

(c-d) Cooling from $160^{\circ} \mathrm{C}$ to the shear temperature of $136{ }^{\circ} \mathrm{C}$ at $10^{\circ} \mathrm{C} / \mathrm{min}\left(T_{\mathrm{m}}\right.$ of HDPE is 129 $\left.{ }^{\circ} \mathrm{C}\right)$;

(d-e) Once the set shear temperature is reached, shear for $100 \mathrm{~s}$ at a fixed rate of $1 / \mathrm{s}$ is applied;

(e-f) Cooling from $136{ }^{\circ} \mathrm{C}$ to $90^{\circ} \mathrm{C}$ at $10^{\circ} \mathrm{C} /$ min and recording WAXD/SAXS patterns every 15

S. 


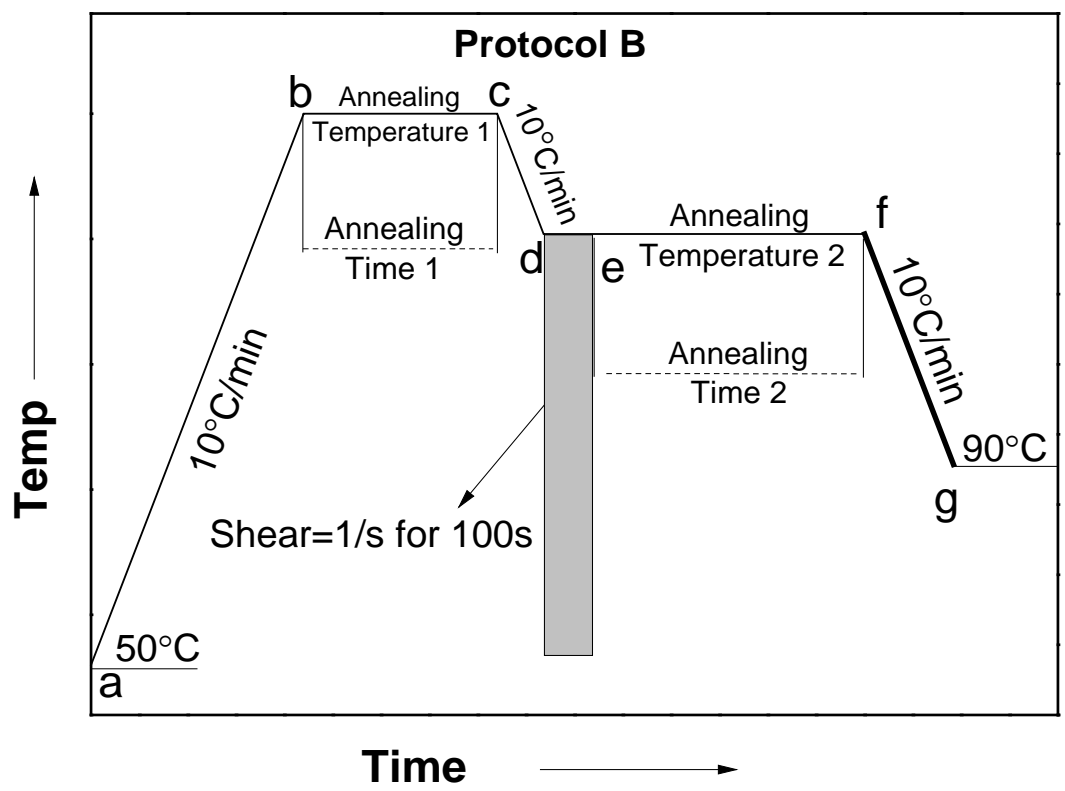

Scheme 2. The schematic representation of shear and temperature profiles during the WAXD/SAXS experiments of Protocol B.

(a-b) Heating from $50{ }^{\circ} \mathrm{C}$ to the annealing temperature of $160{ }^{\circ} \mathrm{C}$ at $10{ }^{\circ} \mathrm{C} / \mathrm{min}$;

(b-c) Annealing at $160{ }^{\circ} \mathrm{C}$ for 5 min with the aim to remove the sample's thermo-mechanical history;

(c-d) Cooling from $160^{\circ} \mathrm{C}$ to the shear temperature of $136^{\circ} \mathrm{C}$ at $10^{\circ} \mathrm{C} / \mathrm{min}$;

(d-e) Once the set shear temperature is reached, shear for $100 \mathrm{~s}$ at a fixed rate of $1 / \mathrm{s}$ is applied;

(e-f) Isothermal annealing at $136{ }^{\circ} \mathrm{C}$ for 10 min after the cycle (d-e);

(f-g) Cooling from $136{ }^{\circ} \mathrm{C}$ to $90^{\circ} \mathrm{C}$ at $10^{\circ} \mathrm{C} / \mathrm{min}$ and recording 2D WAXD/SAXS patterns every $15 \mathrm{~s}$. 


\section{Results and discussion}

\subsection{Morphology of filler}

The exfoliation and oxidation state of GON were characterized by TEM and XRD, Figure 2.
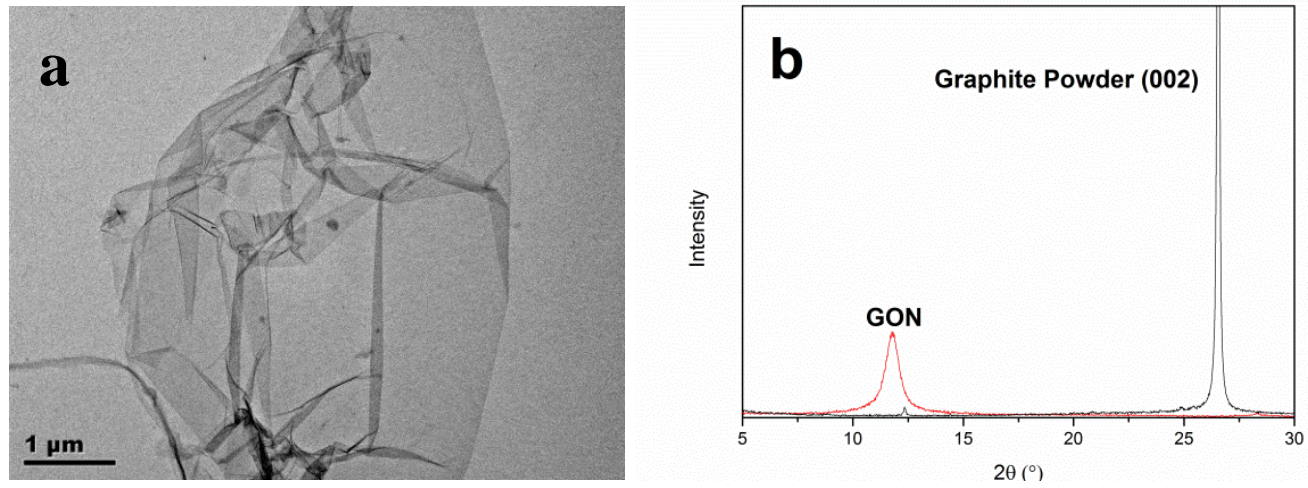

Figure 2. TEM image of a single layer of GON (a), and XRD spectrum of GON film and raw graphite powder (b).

Figure 2a shows an example of an exfoliated GON monolayer suspended on a Cu grid. The monolayer was obtained from its water suspension. Monolayers and few-stacked layers were observed across the whole view. To recall, as described in ref 6, the GON layer was taken from the top layer of the suspension obtained after the washing procedure. Figure $2 \mathrm{~b}$ illustrates shift in $2 \theta$ from $26^{\circ}$ to $11^{\circ}$ in the (002) diffraction peak after oxidation and ultrasonication of GON. The shifted peak, after the oxidation and ultrasonication, shows broadening. These results indicate disruption in the packing of graphite planes after oxidation of the raw graphite.

The thermal reduction of GON at temperature of $160^{\circ} \mathrm{C}$ was investigated by XPS, and the high resolution C1s peaks of GON and rGON are reproduced in Figure 3 and the corresponding data are shown in Table 1 and 2, respectively. 

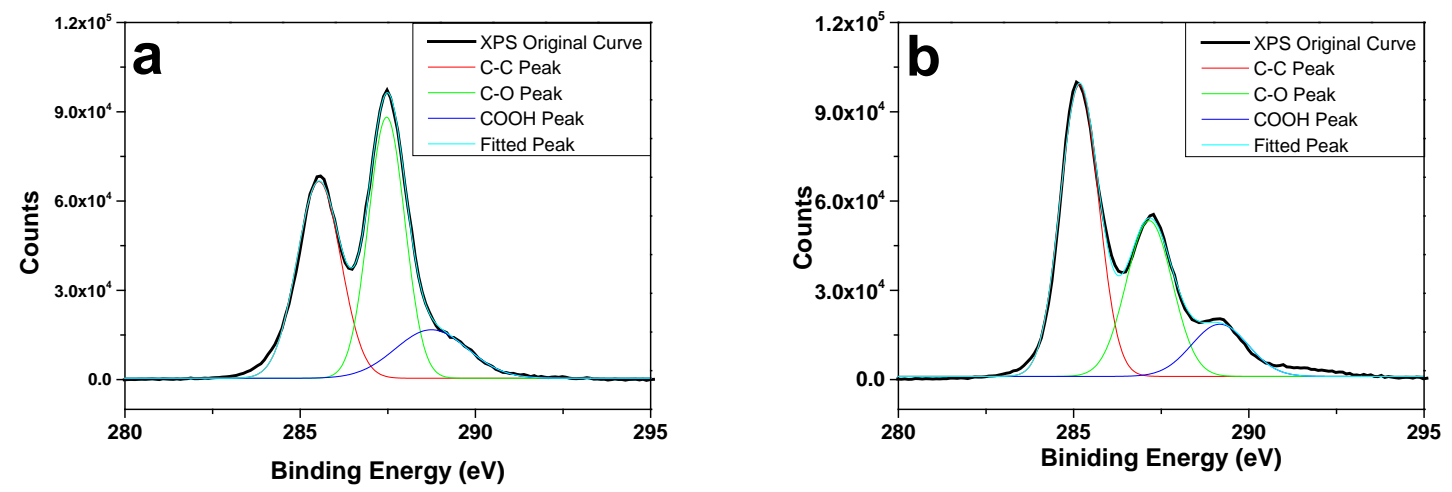

Figure 3 a) high resolution XPS spectra of C1s for GON, and b) high resolution XPS spectra of C1s for rGON; the measurements were carried out on dried GON and rGON films, respectively.

Table 1 XPS data of GON

\begin{tabular}{|l|l|l|l|}
\hline GON & Peak BE (eV) & At. \% & Bond \\
\hline \multirow{3}{*}{ C1s } & 285.08 & 39 & C-C and C=C \\
\cline { 2 - 5 } & 287.16 & 43 & C-O (epoxy, hydroxyl groups) \\
\cline { 2 - 4 } & 289.16 & 18 & carbonyl groups \\
\hline
\end{tabular}

Table 2 XPS data of rGON

\begin{tabular}{|l|l|l|l|}
\hline GON & Peak BE (eV) & At. \% & Bond \\
\hline \multirow{3}{*}{ C1s } & 285.08 & 49 & C-C and C=C \\
\cline { 2 - 5 } & 287.16 & 34 & C-O (epoxy, hydroxyl groups) \\
\cline { 2 - 4 } & 289.16 & 17 & carbonyl groups \\
\hline
\end{tabular}


As observed in Figure 3, Table 1 and Table 2, after thermal reduction the C-C peak increases at the expenses of the C-O peak, indicating successful reduction at temperature of $160{ }^{\circ} \mathrm{C}$. Such reduction at low temperature has also been reported in reference [21].

\subsection{Rheological response}

Linear oscillatory rheology is a well-established technique in the study of polymer-based nanocomposites [31]. Absolute values of the storage ( $\left.G^{\prime}\right)$ and loss ( $G^{\prime}$ ) moduli, as well as their frequency dependence, reveal viscoelastic response of the polymer and influence of filler on polymer matrix.
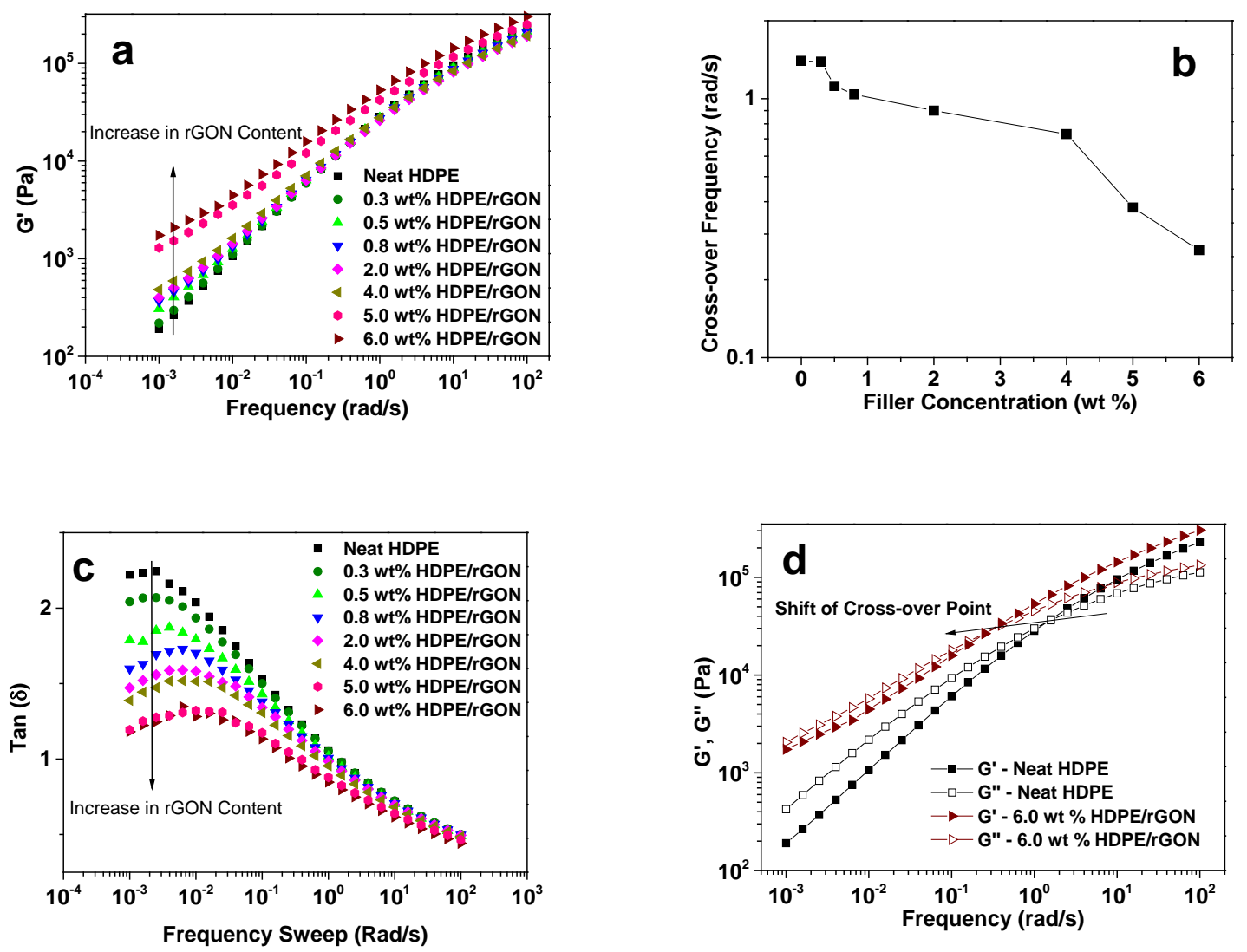

Figure 4. (a) Storage modulus ( $G^{\prime}$ ) of HDPE/rGON composites as a function of frequency; (b) $G^{\prime} / G$ ” cross-over frequencies of HDPE/rGON composites having different filler content; (c) corresponding damping factor tan $(\delta)$, as a function of frequency for HDPE/rGON composites. The phase angle is obtained from the frequency sweep data in (a); (d) shift of cross-over point from plain HDPE to $6.0 \mathrm{wt} \% \mathrm{HDPE} / \mathrm{rGON}$ nanocomposite. The measurements were carried out under isothermal conditions at $160{ }^{\circ} \mathrm{C}$. 
Figure 4a shows storage modulus $\left(G^{\prime}\right)$ of the samples with different filler concentrations as a function of angular frequency. Due to the higher elastic modulus of rGON compared to the polymer melt, the absolute value of $G$ ' increases progressively with increasing filler content, up to $6.0 \mathrm{wt} \%$ at the low frequency range (frequency $<1.0 \mathrm{rad} / \mathrm{s}$ ). The increase of $G$ ' is evident at the

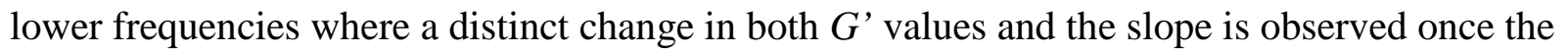
filler content increases from 4.0 to $5.0 \mathrm{wt} \%$. Similar changes have been reported in an earlier study where carbon nanotubes (CNT) composites were investigated [32]. Another indication concerning influence of the filler on the polymer matrix is the $G^{\prime} / G^{\prime}$ cross-over frequency, which reflects the transition from predominantly elastic to viscous behavior in the viscoelastic polymer melt. In a plain polymer melt at equilibrium state, this point is related to the chain constraint renewal time by the so-called reptation dynamics [33]. In Figure 4b, a gradual shift in cross-over point to lower frequencies with the increasing filler content up to $4.0 \mathrm{wt} \%$ is observed. Beyond $4.0 \mathrm{wt} \%$, significant shift in the cross-over frequency to lower values occurs, suggesting that the presence of the filler restrains the relaxation of polymer chains by arresting the chain segments. The dramatic shift in the cross-over frequency from $4.0 \mathrm{wt} \%$ to $5.0 \mathrm{wt} \%$ is attributed to the formation of a solid-like network arising from the close connectivity of rGON, considered as rheological percolation. Similar results from HDPE/CNTs composites have also been reported in earlier studies [32,34,35]. It is further noticed that by increasing the filler concentration, Figure 4c, the decrease in the absolute value of the damping factor $(\tan (\delta)=$ $\left.G^{\prime \prime} / G^{\prime}\right)$ becomes more evident at the low frequency range $(<0.1 \mathrm{rad} / \mathrm{s})$. At a filler concentration of $6.0 \mathrm{wt} \%$, a second cross-over point appears at low frequencies suggesting the formation of a weak network arising from the percolation of rGON sheets in the polymer melt, Figure 4d Similar observations have been reported in the composites of polycarbonate/CNTs, where the 
authors have reported significant increase in $G$ ' at low frequencies and the appearance of a second cross-over point at low frequencies with the increase in concentration of CNT [36].

Figure 5 shows change in the elastic modulus recorded at different frequencies of the composites having different filler concentrations. For comparison, two frequencies above (1.6 and $10 \mathrm{rad} / \mathrm{s})$ and two frequencies below $(0.01$ and $0.001 \mathrm{rad} / \mathrm{s})$ the cross-over point (0.2-1.0 $\mathrm{rad} / \mathrm{s}$ ) of elastic and viscous moduli have been chosen. From the data it is apparent that for the frequencies above the cross-over point the elastic modulus is nearly independent of the filler concentration, whereas the strong influence of filler concentration on modulus at frequencies below the cross-over point, close to the terminal region, is observed. The dependence of the filler concentration on the elastic modulus, below the cross-over point, suggests that due to the interaction of polyethylene chains with rGON, the chain dynamics is influenced to an extent that the viscous response of the viscoelastic polymer melt is strongly affected.

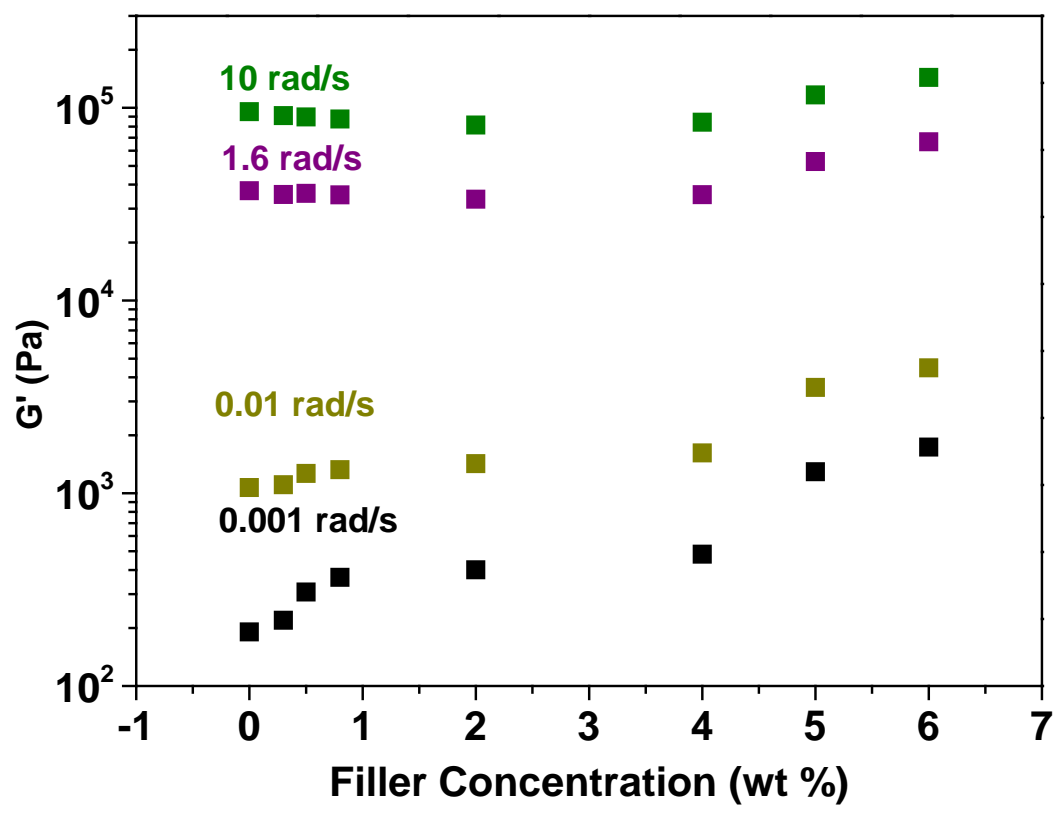


Figure 5. Storage modulus ( $\left.G^{\prime}\right)$ of HDPE/rGON nanocomposites recorded at different frequencies for a range of filler concentrations. Two frequencies of $10 \mathrm{rad} / \mathrm{s}$ and $1.6 \mathrm{rad} / \mathrm{s}$ are above the cross-over point $(0.2-1.0 \mathrm{rad} / \mathrm{s})$, whereas the frequencies of $0.01 \mathrm{rad} / \mathrm{s}$ and $0.001 \mathrm{rad} / \mathrm{s}$ are chosen below the cross-over point. From the data, plotted on the logarithmic scale, it is apparent that the elastic modulus at low frequencies increases with the increase in filler content at low concentrations.

\subsection{Wide angle $X$-ray diffraction on $\mathrm{HDPE} / \mathrm{rGON}$ composites under flow conditions}

It is well established that flow plays a crucial role in the extension and orientation of polymer chains, resulting in the formation of highly anisotropic, oriented structures upon crystallization $[37,38]$. Nanofillers are known to have a noticeable influence on chain extension and orientation influencing relaxation dynamics. The non-equilibrium, constrained melt serves as precursors for crystallization. To have insight on the influence of rGON on the polymer melt after application of non-linear shear, we monitored the Herman's orientation factor of the samples by WAXD following Protocols A and B depicted in Schemes 1 and 2, respectively. The difference in the schemes is on the annealing time after the application of shear in melt. The orientation of polymer crystals are estimated by calculating Herman's orientation factor $f_{\mathrm{h}}$ using equation (1) [39],

$$
f_{h}=\frac{1}{2}\left\{3<\cos ^{2} \emptyset>-1\right\} \quad \text { Equation (1) }
$$

Where $f_{\mathrm{h}}$ is the Herman's orientation factor, $\left\langle\cos ^{2} \emptyset\right\rangle$ is the average cosine squared weighted by the intensity as a function of the radial angle for the oriented crystallographic planes and it can be calculated from equation (2) [39],

$$
<\cos ^{2} \emptyset>=\frac{\int_{0}^{90} I(\theta) \cos ^{2} \theta \sin \theta d \theta}{\int_{0}^{90} I(\theta) \sin \theta d \theta}
$$

where $I(\theta)$ is the intensity of the planes normal to $c$-axis and $\theta$ is the azimuthal angle.

Herman's orientation factor gives a good qualitative description of the average crystal plane 
orientation. The value of $f_{\mathrm{h}}$ may vary between -0.5 and 1.0 for perpendicular and parallel crystal plane orientation with respect to the flow direction, respectively.

Figure 6a and Figure 6b illustrate the Herman's orientation factor for diffraction planes (110) and (200) of orthorhombic polyethylene crystals. The crystallization starts when the factor deviates from zero. These values are determined by integrating the diffraction patterns recorded on following the Scheme 1, where the samples were cooled immediately after the application of shear. From the figures it is apparent that the diffraction planes (110) and (200) exhibit the flow induced orientation for both the neat polymer and the composites. However, the absolute value of the orientation factor in the composites is higher than that in the neat sample and it becomes more pronounced with the increasing rGON concentration, indicating that the presence of rGON enhances the orientation during flow.
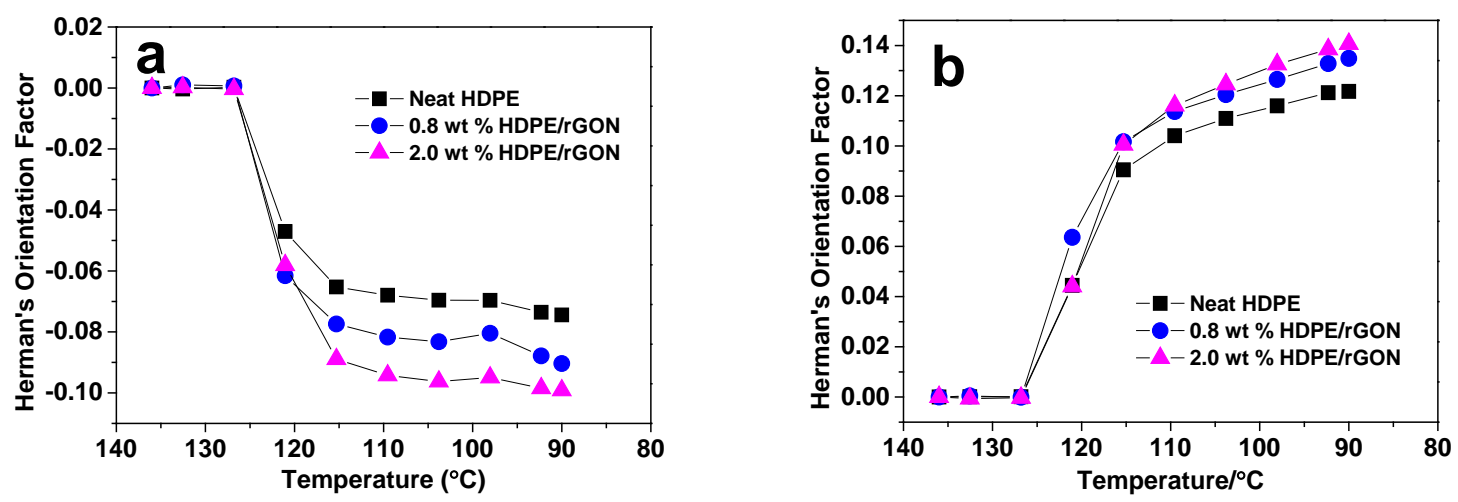

Figure 6. Herman's orientation factor $f_{\mathrm{h}}$ of polyethylene's diffraction planes, (110) (a) and (200) (b) as a function of temperature recorded on cooling, with and without rGON, after the application of shear (Scheme 1).

In the neat sample the cause of the orientation can be attributed to higher relaxation time of the higher molar mass component compared to the low molar mass component in the sample having broad polydispersity. Most of the low molar mass polymer chains in the neat sample tend to relax after the application of shear. The reptation time determined from the cross-over point of 
the sample is approximately $1 \mathrm{~s}$, Figure $4 \mathrm{~b}$. The difference in the relaxation times of PE chains having different molar mass contributes to the anisotropic structure of the crystallized material, Figure 6. However, the enhancement of anisotropy in the composites is dependent on the filler content. After shear, the mobility of chains is highly restricted by the filler-chain interaction. With increasing filler concentration, more polymer chains are hindered and hence the orientation becomes more pronounced. The corresponding WAXD patterns of the samples recorded at $90{ }^{\circ} \mathrm{C}$ after cooling are illustrated in Figure 7 where anisotropic rings of (110) and (200) diffraction planes are observed in neat as well as its composite samples. The inner (110) diffraction plane shows equatorial arcs representing chain orientation along the flow direction. The outer (200) diffraction plane shows meridian arcs indicating crystal plane orientation perpendicular to the flow direction.

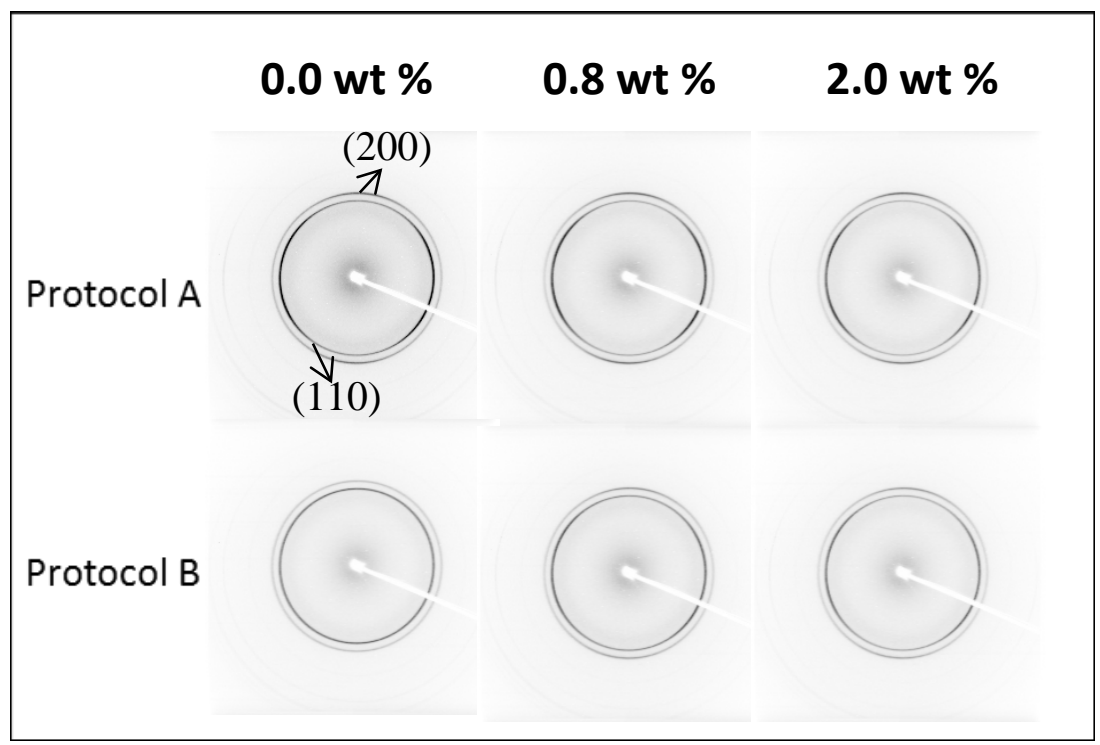

Figure 7. WAXD patterns of the HDPE samples recorded at $90{ }^{\circ} \mathrm{C}$ following the protocols depicted in Scheme 1 and Scheme 2, respectively.

To follow the influence of annealing time on relaxation, after the application of shear, Protocol B depicted in Scheme 2 is followed. The samples are left to anneal under isothermal condition, at $136{ }^{\circ} \mathrm{C}$ for $10 \mathrm{~min}$, after the application of shear. Figure 8a and Figure 8b depict the Herman’s 
orientation factors of neat HDPE and its composites. It is evident from the figures that the orientation factor for neat HDPE is close to zero, which represents the isotropic state, whereas the composites show non-zero values that become more pronounced with increasing the filler concentration.
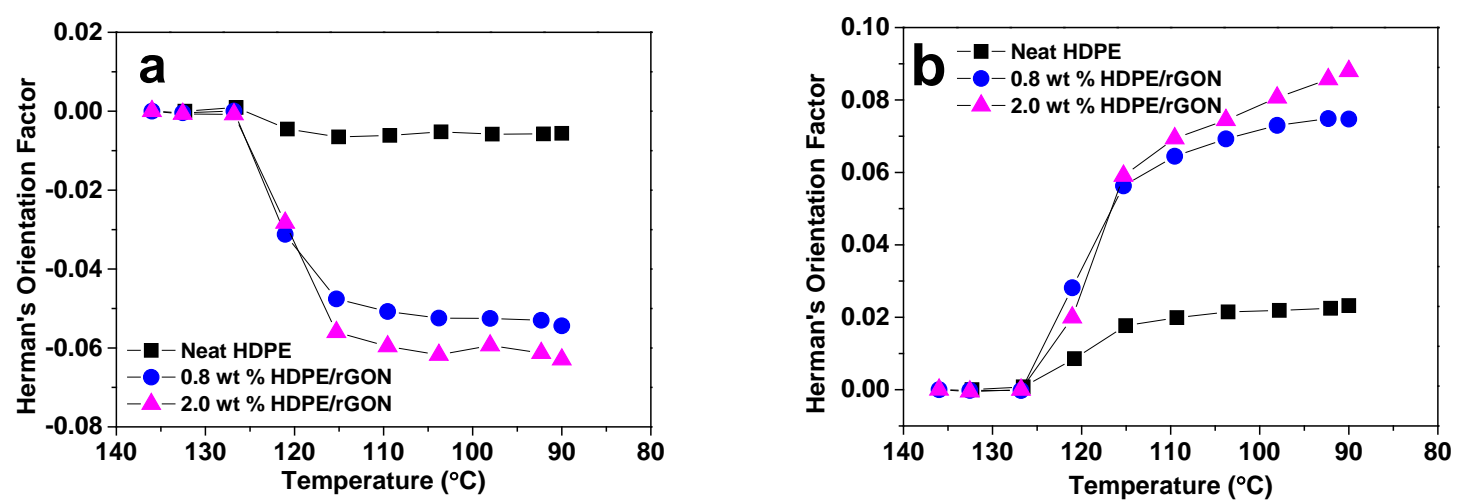

Figure 8. Herman's orientation factor $\left(f_{\mathrm{h}}\right)$ of polyethylene's (110) diffraction plane (a), and (200) diffraction plane (b) as a function of temperature during cooling, with and without rGON, obtained on following the protocol B depicted in Scheme 2.

Figure $4 \mathrm{~b}$ shows the cross-over point for neat HDPE from elastic to viscous behavior at $\sim 1.0$ $\mathrm{rad} / \mathrm{s}$, i.e. the relaxation time for most of the chains is approximately $1 \mathrm{~s}$. Thus the $10 \mathrm{~min}$ of the isothermal annealing time after application of shear at $136^{\circ} \mathrm{C}$ is likely to be long enough for the relaxation of chains in neat HDPE. On the contrary, using the same protocol in the presence of the filler, considerable orientation is maintained. These observations are in agreement with the observed shift in the cross-over point in the presence of filler reported in Figure 4b, confirming that chains having interaction with the filler are unable to adopt the random coil conformation and maintain orientation even after isothermal annealing in melt for $10 \mathrm{~min}$. In addition, the obvious orientation of the chains in the presence of filler may arise from the adsorption of HDPE chains on rGON that leads to significant chain stretching in a shear field. These adsorbed oriented chains contribute to the formation of non-equilibrium oriented domains, which enhances 
crystallization followed by the development of highly anisotropic structure. The influence of the filler concentration on chain orientation is evident. Their corresponding 2D patterns recorded at $90{ }^{\circ} \mathrm{C}$, after the shear, isothermal treatment and crystallization, are shown in Figure 7 where the neat sample shows an isotropic pattern whereas the composites show orientation for both (110) and (200) diffraction planes, indicating chain orientation due to strong chain-filler interaction that hinders the relaxation of chains.

\subsection{The Deborah number}

To quantify the effect of flow on the microscopic chain configuration and to understand whether there is significant chain stretch occurring during applied shear that could cause flow induced crystallization [38], the Deborah number accounting for the chain stretch relaxation and reorientation processes is calculated. Chain stretching refers to configurations where the length of the occupied tube departs from its equilibrium state [40], while reorientation occurs by reptation dynamics.

The Deborah number proposed by Reiner, describes a non-dimensional number that distinguishes how a particular material behaves within a given time scale [41], as shown in Equation (3).

$$
D e=\frac{t_{r e l}}{t_{o b s}}
$$

where $t_{\text {rel }}$ refers to time required for material to relax and $t_{\mathrm{obs}}$ refers to the time of observation. De has been applied under shear flow to investigate the crystallization behavior of stretched chains in flow induced crystallization (FIC) [38]. For this purpose, two time scales, $\tau_{\text {rep }}$ and $\tau_{\text {s, }}$ have been proposed to quantify the orientation and stretch using two Deborah numbers described in equations (4) and (5), for the applied shear rate. Where $\tau_{\text {rep }}$ refers to longest reptation time, 
terminal relaxation time, and $\tau_{\mathrm{s}}$ refers to chain retraction time associated with the relaxation time of the chain retraction,

$$
\begin{gathered}
D e_{\text {rep }}=\tau_{\text {rep }} \bullet \dot{\gamma} \\
D e_{\mathrm{s}}=\tau_{\mathrm{s}} \dot{\gamma}
\end{gathered}
$$

Likhtman and McLeish proposed a relationship between terminal relaxation time, $\tau_{\text {rep }}$, and disengagement time in the absence of fluctuations, $\tau_{\mathrm{d}}[42]$ :

$$
\frac{\tau_{r e p}}{\tau_{d}}=1-\frac{3.38}{Z^{0.5}}+\frac{4.17}{Z}-\frac{1.55}{Z^{1.5}}
$$

where $\tau_{\mathrm{d}}$ is given by $3 \mathrm{Z}^{3} \tau_{\mathrm{e}}, \mathrm{Z}$ is the number of entanglements per chain, $\mathrm{Z}=M_{\mathrm{w}} / M_{\mathrm{e}}$, of high molecular weight chains (HMW) with $M_{\mathrm{e}}=828 \mathrm{~g} / \mathrm{mol}$ [43] and $M_{\mathrm{w}}=2.6 \times 10^{5} \mathrm{~g} / \mathrm{mol}$, Figure 1 . Equilibrium time at $136{ }^{\circ} \mathrm{C}, \tau_{\mathrm{e}}=1.75 \times 10^{-8} \mathrm{~s}$, is extrapolated from the equilibrium state at $190{ }^{\circ} \mathrm{C}$ $\left(7 \times 10^{-9} \mathrm{~s}\right)$ using time-temperature superposition principle of Arrhenius dependence empirical expression with activation energy, $E_{\mathrm{a}}=26.7 \mathrm{~kJ} / \mathrm{mol}$ at $160^{\circ} \mathrm{C}$ [44]. The retraction time, $\tau_{\mathrm{s}}$, is obtained by $\mathrm{Z}^{2} \tau_{\mathrm{e}}{ }^{33}$ The values of $\mathrm{Z}$ and $\tau_{\mathrm{d}}$ are given below:

$$
\begin{gathered}
\mathrm{Z}=\frac{M_{w}}{M_{e}}=\frac{2.5 \times 10^{5}}{828} \approx 302 \\
\tau_{d}=3 \times \mathrm{Z}^{3} \times \tau_{e} \approx 1.4 \mathrm{~s}
\end{gathered}
$$

The calculated values of $\tau_{\text {rep }}, \tau_{\mathrm{s}}$ and their $D e$, at $136{ }^{\circ} \mathrm{C}$, are reported in Table 3 .

Table 3. - The calculated relaxation times and the Deborah numbers of HMW chains

\begin{tabular}{|c|c|c|c|c|}
\hline Shear Rate (/s) & $\tau_{\text {rep }}(\mathrm{s})$ & $\tau_{\mathrm{s}}(\mathrm{s})$ & $D e_{(\text {rep })}$ & $D e_{(\mathrm{s})}$ \\
\hline 1 & 1.2 & 0.002 & 1.2 & 0.002 \\
\hline
\end{tabular}


As reported in literature, in principle, significant chain stretch should occur when $\tau_{\mathrm{s}} \times \dot{\gamma}>1$ [40], and this is considered an essential condition for the formation of shish-kebab precursor and thus shish-kebab structure after crystallization $[38,45]$. In the case of shear rate of $1 / \mathrm{s}, D e_{(\mathrm{s})}<1$, only insignificant stretch can occur during shear. These results explain the absence of shish-kebab structure formation on the application of mild shear 1/s. In Figure 9, 2D SAXS patterns of plain HDPE and its composites are shown. These patterns, following Scheme 1, were recorded at 136 ${ }^{\circ} \mathrm{C}$ just after the application of shear and at $90{ }^{\circ} \mathrm{C}$ on cooling to the crystallization temperature.
0.0 wt \%
0.8 wt $\%$
$2.0 \mathrm{wt} \%$
$136{ }^{\circ} \mathrm{C}$

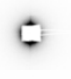
$90{ }^{\circ} \mathrm{C}$
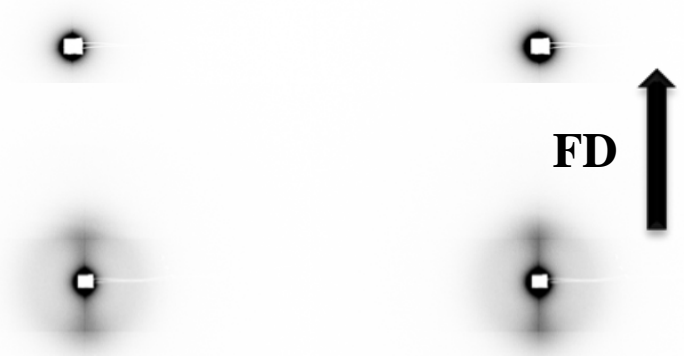
Figure 9. 2D SAXS patterns of samples recorded at $136{ }^{\circ} \mathrm{C}$ (after the application of shear) and $90{ }^{\circ} \mathrm{C}$ (after the dynamic cooling) following the Protocol A described in Scheme 1. The samples measured are neat sample, $0.8 \mathrm{wt} \%$ and $2.0 \mathrm{wt} \%$ HDPE/rGON composites. The 1/s shear was applied for $100 \mathrm{~s}$ at $136{ }^{\circ} \mathrm{C}$. Just after the shear the samples were cooled to $90{ }^{\circ} \mathrm{C}$, at $10^{\circ} \mathrm{C} / \mathrm{min}$.
From SAXS patterns, recorded at $136^{\circ} \mathrm{C}$, the absence of shish structure suggests that the sample experienced mild stretch during shear. The SAXS data recorded at $90{ }^{\circ} \mathrm{C}$, following the Scheme 1, is in accordance with WAXD data, confirming increase in oriented structure with increasing filler concentration, as shown in Figures 6 and 7. These findings are in agreement with the rheological response of the materials depicted in Figure $4 \mathrm{~b}$ where shift in the cross-over point to lower frequencies with increasing filler content is observed, indicating the increase in the effective reorientation time with the increasing filler concentration. 
It is important to notice that the quantitative calculation of the Deborah number is strictly valid for the neat polymer, because $\tau_{\mathrm{e}}$ applied for the calculation is based on the polymer without any filler. The presence of graphene is likely to influence chain dynamics at different frequencies. However, the relaxation moduli $G$ ' and $G$ " in Figure 4 and Figure 5, show no significant changes in the high frequency range ( $\geq 0.1 \mathrm{rad} / \mathrm{s}$ - i.e. higher than the cross-over frequencies) indicating that for the filler concentrations below $4.0 \mathrm{wt} \%$, chain stretch relaxation is little influenced, and hence the calculated Deborah Number for the composites should not depart significantly from the true values.

\section{Conclusions}

Reduced graphene oxide nanoplatelets (rGONs)/high density polyethylene (HDPE) nanocomposites were prepared using a two-step solution/suspension method. The rheological percolation measured by linear oscillatory rheology reveals a threshold at $4.0 \mathrm{wt} \%$. Time resolved WAXD/SAXS investigation on the HDPE/rGON samples subjected to shear flow depicts a strong chain-filler interaction that maintains strong chain orientation. On immediate cooling, after the application of shear following the Scheme 1, polymer chains in the composites show stronger orientation compared to the neat sample, whereas on following the Scheme 2, after the application of shear when the sample is left to anneal at higher temperatures, no significant orientation is observed. However, on cooling to room temperature the composite shows retention of orientation equivalent to that observed in the sample cooled directly from melt after the application of shear following the Scheme 1 . These findings, supported by the rheological response of the neat and the composite samples, demonstrate strong interaction between rGON and polyethylene chains. The Deborah number is calculated to understand the 
influence of applied shear on chain stretching, which suggests that in the applied shear conditions the investigated samples experienced mild chain stretch, resulting in the absence of shish formation. Thus the orientation observed in the composites at room temperature is primarily attributed to the interaction of rGON with chain segments. Similar to our earlier findings on UHMWPE/rGON composite where the maximum drop in viscosity as a function of concentration is observed at $0.8 \mathrm{wt} \%$ of rGON at the frequency of $10 \mathrm{rad} / \mathrm{s}$ (Figure 10a) [6], in HDPE/rGON samples, the maximum drop in viscosity is observed at much higher frequency of $100 \mathrm{rad} / \mathrm{s}$ (Figure 10b) at rGON content between 2.0 to $4.0 \mathrm{wt} \%$, Figure 10b. The difference in the frequency and the filler concentration for the phenomenon is attributed to the difference in the chain length. The comparison is depicted in Figure 10. The drop in the storage modulus is attributed to the adhesion of longer chains in the polymer matrix to the filler surface, whereas increase in the modulus with the increasing concentration of rGON is attributed to the clustering of rGON.
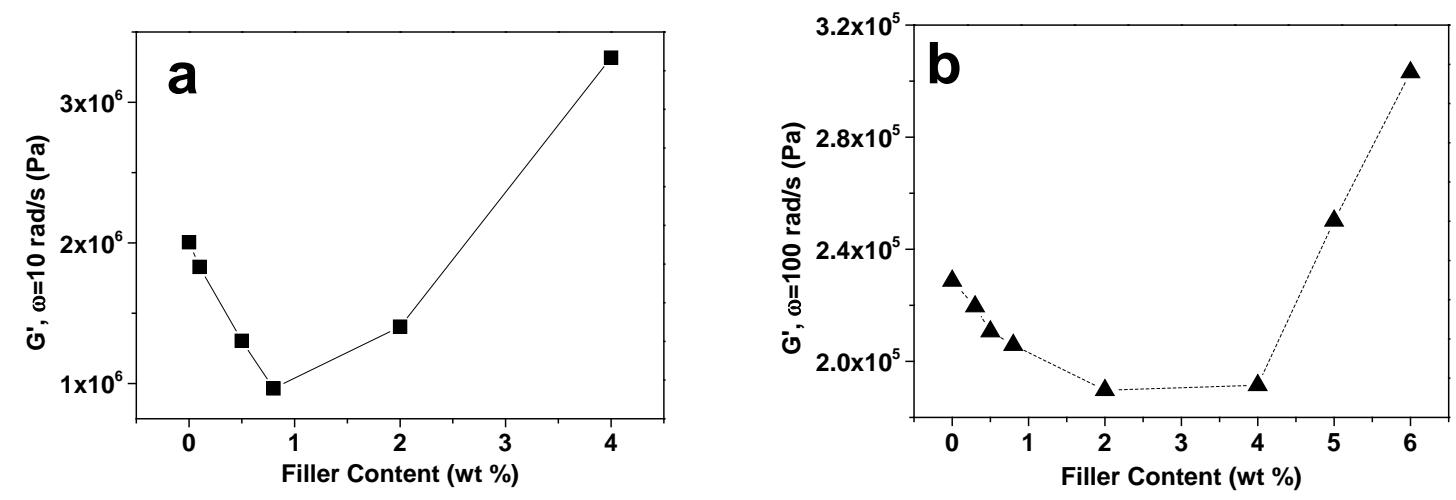

Figure 10. a) Storage modulus of UHMWPE/rGON composites at rubbery plateau (10 rad/s) of frequency sweep [6], and b) storage modulus of HDPE/rGON composites at frequency of 100 $\mathrm{rad} / \mathrm{s}$. 


\section{AUTHOR INFORMATION}

\section{Corresponding Authors:}

*(S.R.) E-mail: S.Rastogi@lboro.ac.uk; *(S.R.) E-mail: S.Ronca@lboro.ac.uk

\section{ACKNOWLEDGEMENT}

The authors wish to acknowledge financial support provided by Loughborough University, UK. Efren Andablo Reyes acknowledges financial support from the Dutch Polymer Institute, The Netherlands.

\section{References}

[1] Paul, D. R.; Robeson, L. M. Polymer nanotechnology: nanocomposites. Polymer 2008, 49, 3187-3024.

[2] Sahoo, N. G.; Rana, S.; Cho, J. W.; Li, L.; Chan, S. H. Polymer nanocomposites based on functionalized carbon nanotubes. Prog. Polym. Sci. 2010, 35, 837-867.

[3] Medellin-Rodriguez, F. J.; Burger, C.; Hsiao, B. S.; Chu, B.; Vaia, R.; Philips, S. Timeresolved shear behavior of end-tethered nylon 6-clay nanocomposites followed by nonisothermal crystallization. Polymer 2001, 42 (21), 9015-9023.

[4] Fu, B. X.; Yang, L.; Somani, R. H.; Zong, S. X.; Hsiao, B. S.; Philips, S.; Blanski, R.; Ruth, P. Crystallization studies of isotactic polyethylene containing nanostructured polyhedral oligomeric silsesquioxane molecules under quiescent and shear conditions. J. Polym. Sci. Part B: Polym. Phys. 2001, 39 (22), 2727-2739. 
[5] Zhang, Q.; Lippits, D. R.; Rastogi, S. Dispersion and rheological aspects of SWNTs in ultrahigh molecular weight polyethylene. Macromolecules 2006, 39(2), 658-666.

[6] Liu, K.; Ronca, S.; Andablo-Ryes, E.; Forte, G.; Rastogi, S. Unique rheological response of Ultrahigh Molecular Weight Polyethylenes in the presence of reduced graphene oxide. Macromolecules 2014, 48 (1), 131-139.

[7] Li, Y.; Kroger, M.; Liu, K. W. Nanoparticle effect on the dynamics of polymer chains and their entanglement network. Phys. Rev. Lett. 2012, 109, 118001.

[ 8 ] Rastogi, S.; Lippits, D. R.; Peters, G. W. M.; Graf, R.; Yao, Y.; Spiess, H. W. Heterogeneity in polymer melts from melting of polymer crystals. Nat. Mater. 2005, 4, 635-641.

[9] Kharchenko, S. B.; Douglas, J. F.; Obrzut, J.; Grulke, E. A.; Migler, K. B. Flow-induced properties of nanotube-filled polymer materials. Nat. Mater. 2004, 3, 564-568.

[10] Jain, S.; Goosen, G. W. M.; Van Duin, M.; Lemstra, P. J. Strong decrease in viscosity of nanoparticle-filled polymer melts through selective adsorption. Soft Matter 2008, 4, 1848-1854.

[11] Patil, N.; Balzano, L.; Portale, G.; Rastogi, S. Influence of shear in the crystallization of polyethylene in the presence of SWNTs. Carbon 2010, 48, 4116-4128.

[12] Balzano, L. Flow Induced Crystallization of Polyolefins. PhD Thesis. Eindhoven University of Technology, 2008.

[13] Kimata, S.; Sakurai, T.; Nozue, Y.; Kasahara, T.; Yamaguchi, N.; Karino, T.; Shibayama, M.; Kornfield, J. A. Molecular basis of the shish-kebab morphology in polymer crystallization. Science 2007, 316, 1014-1017. 
[14] Novoselov, K. S.; Geim, A. K.; Morozov, S. V.; Jiang, D.; Zhang, Y.; Dubonos, S. V.; Jiang, D.; Zhang, Y.; Dubonos, S. V.; Grigorieva, I. V.; Firsov, A. A. Electric field effect in atomically thin carbon films. Science 2004, 306, 666-669.

[15] Nilsson, J.; Neto, A. H. C.; Guinea, F.; Peres, N. M. R. Electronic properties of graphene multilayers. Phys. Rev. Lett. 2006, 97, 266801.

[16] Balandin, A. A.; Ghosh, S.; Bao, W. Z.; Calizo, I.; Teweldebrhan, D.; Miao, F.; Lau, C. N. Superior thermal conductivity of single-layer Graphene. Nano Lett. 2008, 8 (3), 902-907.

[17] Lee, C. G.; Wei, X. D.; Kysar, J. W.; Hone J. Measurement of the elastic properties and instrinsic strength of manolayer graphene. Science 2008, 231, 385-388.

[18] Krompiewski, S. Electronic transport through side-contacted graphene nanoribbons: effects of overlap, aspect ratio and orientation. Nanotechnology 2011, 22, 445201.

[19] Stoller, M. D.; Park, S.; Zhu, Y.; An, J.; Ruoff, R. S. Graphene-Based Ultracapacitors. Nano Lett. 2008, 8 (10), 3498-3502.

[20] Hu, H. L.; Zhang, G.; Xiao, L.; Wang, H.; Zhang, Q.; Zhao, Z. Preparation and electrical conductivity of graphene/ultrahigh molecular weight polyethylene composites with a segregated structure. Carbon 2012, 12, 4596-4599.

[21] Chen, W.; and Yan, L. Preparation of graphene by a low-temperature thermal reduction at atmosphere pressure. Nanoscale 2010, 2, 559-563. 
[22] Dykes, L. M. C.; Torkelson, J. M.; Burghardt, W. R.; Krishnamoorti, R. Shear-induced orientation in polymer/clay dispersions via in situ X-ray scattering. Polymer 2010, 51, 49164927.

[23] Schmidt, G.; Nakatani, A. I.; Butler, P. D.; Karim, A.; Han, C. C.; Shear orientation of viscoelastic polymer-clay solutions probed by flow birefringence and SANS. Macromolecules 2000, 30(20), 7219-7222.

[24] Schmidt, G.; Nakatani, A. I., Butler, P. D., Han, C. C. Small angle neutron scattering from viscoelastic polymer-clay solutions. Macromolecules 2002, 35, 4725-4732.

[25] Lin-Gibson, S.; Kim, H.; Schmidt, G.; Han, CC.; Hobbie, E. K. Shear-induced structure in polymer-clay nanocomposite solutions. J. Colloid Interface Sci. 2004, 274, 515-525.

[26] Li, L.; Li, B.; Hood, M. A.; Li, C. Y. Carbon nanotubes induced polymer crystallization: the formation of nanohybrid shish-kebabs. Polymer 2009, 50, 953-965.

[27] Liu, P.; Gong, K.; Xiao, P.; Xiao, M. Preparation and characterization of poly(vinyl acetate)-intercalated graphite oxide nanocomposite. J. Mater. Chem. 2000, 10, 933-935.

[28] Mead, D. Determination of molecular weight distributions of linear flexible polymers from linear viscoelastic material functions. J. Rheol. 1994, 38, 1797-1827.

[29] Talebi, S.; Duchateau, R.; Rastogi, S.; Kaschta, J.; Peters, G. W. M.; Lemstra, P. J. Molar mass and molecular weight distribution determination of UHMWPE synthesized using a living homogeneous catalyst. Macromolecules 2010, 43, 2780-2788.

[30] Tervoort-Engelen, Y. M. T.; Lemstra, P. J. Morphology of nascent ultrahigh-molecularweight polyethylene reactor powder: chain-extended versus chain-folded crystals. Polym. Commun. 1991, 32, 343-345. 
[31] Krishnamoorti, R.; Chatterjee, T. Rheology and processing of polymer nanocomposites, in applied polymer rheology: polymer fluids with industrial applications (ed M. Kontopoulou). John Wiley\&Sons, inc, Hoboken, NJ, USA 2011.

[32] Zhang, Q.; Rastogi, S.; Chen, D.; Lipptis, D. R.; Lemstra, P. J. Low percolation threshold in single-walled carbon nanotube/high density polyethylene composites prepared by melting processing technique. Carbon 2006, 44, 778-785.

[33] Doi, M.; Edwards, S. F. The theory of polymer dynamics. Clarendon Press 1986, Oxford.

[34] Valentino, O.; Sarno, M.; Rainone, N. G.; Nobile, M. R.; Ciambelli, P.; Neitzert, H. C.; Simon, G. P. Influence of the polymer structure and nanotube concentration on the conductivity and rheological properties of polyethylene/CNT composites. Physica E 2008, 40, 2440-2445.

[35] McNally, T.; Potschke, P.; Halley, P.; Murphy, M.; Martin, D.; Bell, S. E. J.; Brennan, G. P.; Bein, D.; Lemoine, P.; Quinn, J. P. Polythylene multiwalled carbon nanotube composites. Polymer 2005, 46, 8222-8232.

[36] Mun, S. C.; Kim, M.; Prakashan, K.; Jung, H. J.; Son, Y.; Park, O. O. A new approach to determine rheological percolation of carbon nanotubes in microstructured polymer matrices. Carbon 2014, 67, 64-71.

[37] Somani, R. H.; Hsiao, B. S.; Nogales, A.; Srinivas, S.; Tsou, A. H.; Sics, .;, Balta-Calleja, F. J.; Ezquerra, T. A. Structure development during shear flow-induced crystallization of i-PP: in-situ small-angle X-ray scattering study. Macromolecules 2000, 33(25), 9385-9394. 
[ 38 ] Balzano, L.; Kukalyekar, N.; Rastogi, S.; Peters, G. W. M.; Chadwick, J. C. Crystallization and dissolution of flow-induced precursors. Phys. Rev. Lett. 2008, 100, 048302.

[39] Alexander, L. E. X-ray diffraction methods in polymer science. New York: WileyInterscience: 1969.

[40] Graham, R. S.; Likhtman, A. E.; Mcleish, T. C. B.; Milner, S. T. Microscopic theory of linear, entangled polymer chains under rapid deformation including chain stretch and convective constraint release. J. Rheol. 2003, 47 (5), 1171-1200.

[41] Reiner, M. The Deborah number. Physics Today 1964, 62.

[42] Likhtman, A. E.; McLeish, T. C. B. Quantitative theory for linear dynamics of linear entangled polymers. Macromolecules 2002, 35, 6332-6343.

[43] Fetters, L.J.; Lohse, D. J.; Richter, D.; Witten, T. A.; Zirkel, A. Connection between polymer molecular weight, density, chain dimensions, and melt viscoelastic properties. Macromolecules 1994, 27(17), 4639-4647.

[44] Ngai, K. L.; Plazek, D. J. Relation of internal rotational isomerism barriers to the flow activation energy of entangled polymer melts in the high-temperature Arrhenius region. $J$. Polym. Sci: Polym. Phys. 1985, 23, 2159-2180.

[45] Patil, N.; Balzano, L.; Portale, G.; Rastogi, S. A study on the chain-particle interaction and aspect ratio of nanoparticles on structure development of a linear polymer. Macromolecules 2010, 43 (16), 6749-6759. 
